\title{
Peripheral T-cell Lymphoma of the Uvula: A Rare Entity
}

Fariba Binesh ${ }^{1}$, Hassanali Vahedian Ardakani ${ }^{2}$, Seyed Hossein Shahcheraghi ${ }^{3,4, *}$

${ }^{1}$ Department of Pathology, Shahid Sadoughi University of Medical Sciences and Health Services, Yazd, Iran

${ }^{2}$ Department of Oncology, Shahid Sadoughi University of Medical Sciences and Health Services, Yazd, Iran

${ }^{3}$ Department of Modern Sciences and Technologies, Faculty of Medicine, Mashhad University of Medical Sciences, Mashhad, Iran

${ }^{4}$ Infectious Diseases Research Center, Shahid Sadoughi Hospital, Shahid Sadoughi University of Medical Sciences, Yazd, Iran

*Corresponding author: Seyed Hossein Shahcheraghi, Infectious Diseases Research Center, Shahid Sadoughi Hospital, Shahid Sadoughi University of Medical Sciences, Yazd, Iran. Tel: +989132531389; Fax: +983538224100; E-mail:

DOI: $10.30699 /$ acadpub.mci.3.1.32 shahcheraghih@gmail.com

Submitted: 7 October 2018

Revised: 29 October 2018

Accepted: 21 November 2018

e-Published: 1 January 2019

\section{Keywords:}

Lymphoma

Uvula

Otolaryngology

Pathology

\section{Abstract}

Introduction: Extra-nodal exposition of T-cell non-hodgkin lymphoma (NHL) is extremely rare, and is frequently observed in patients with immune system dysfunction. Peripheral T-cell lymphoma does not express $\mathrm{CD}^{56-}$ is rare. Malignant lymphoma of the uvula is exceedingly unusual. The current study reported a case of peripheral T-cell lymphoma of the uvula in an immunecompetent patient.

Case presentation: The current study reported a case of peripheral T-cell lymphoma of the uvula in a 54-year-old male patient. The diagnosis was based on excisional biopsy and ancillary techniques. The patient was referred to the hemato-oncology ward for treatment.

Conclusion: Considering the fact that the presentation of peripheral T-cell NHL in uvula is uncommon, a high index of suspicion is necessary to diagnose this grave disease. To the best of authors' knowledge, this case is the third case of T-cell lymphoma of the uvula in the English literature.

(C) 2019. Multidisciplinary Cancer Investigation

\section{INTRODUCTION}

Lymphomas are heterogeneous neoplasms determined by the multiplication of lymphoid cells including hodgkin lymphoma (HL) and non-hodgkin lymphoma (NHL) [1]. NHL comprises of B-cell, T-cell, or natural killer (NK)/T-cell subtypes. NHL occurs more generally than HL, and B-cell lymphomas are more common than T-cell lymphomas [1]. NHL of the oral cavity is observed in only $2 \%$ of cases [1]. Various oral subsites of T-cell lymphoma consists of lip, gingiva, palate, maxilla, buccal mucosa, tongue, and uvula [2] Since T-cell lymphoma presents infrequently in oral cavity, it should be placed in differential diagnoses list of any mass in the oral cavity. Physicians meet difficulties in the correct management of this disorder. Histopathology and immunohistochemistry have a critical role in proper diagnosis. The current study reported a rare case of T-cell lymphoma of the uvula in an immune-competent patient. 


\section{CASE PRESENTATION}

A 54-year-old male patient referred to the ear, nose, and throat (ENT) outpatient clinic in August 2016 with progressively worsening odynophagia and sore throat over the past six months. The pain radiated to both ears, and it was accompanied by hoarseness, dysphagia, and loss of appetite. Weight loss (13 $\mathrm{kg}$ ) within the last two months was noticeable. His past medical history was unremarkable. Clinical examination demonstrated uvular swelling with a smooth surface and relatively hard consistency. There were no other abnormal physical findings. He received corticosteroid and antibiotics with partial response. Three weeks later, he was readmitted due to the intensification of his symptoms. Physical examination revealed the previous findings. Due to the persistence of the symptoms, the patient underwent uvulectomy with the clinical suspicion of squamous cell carcinoma (SCC) of the uvula.Histological assessment of the uvula demonstrated focal areas of necrosis with a diffuse pattern of proliferation of relatively small- to medium-sized lymphoid cells. The neoplastic cells had scanty cytoplasm with mild nuclear membrane convolution (Figure 1).
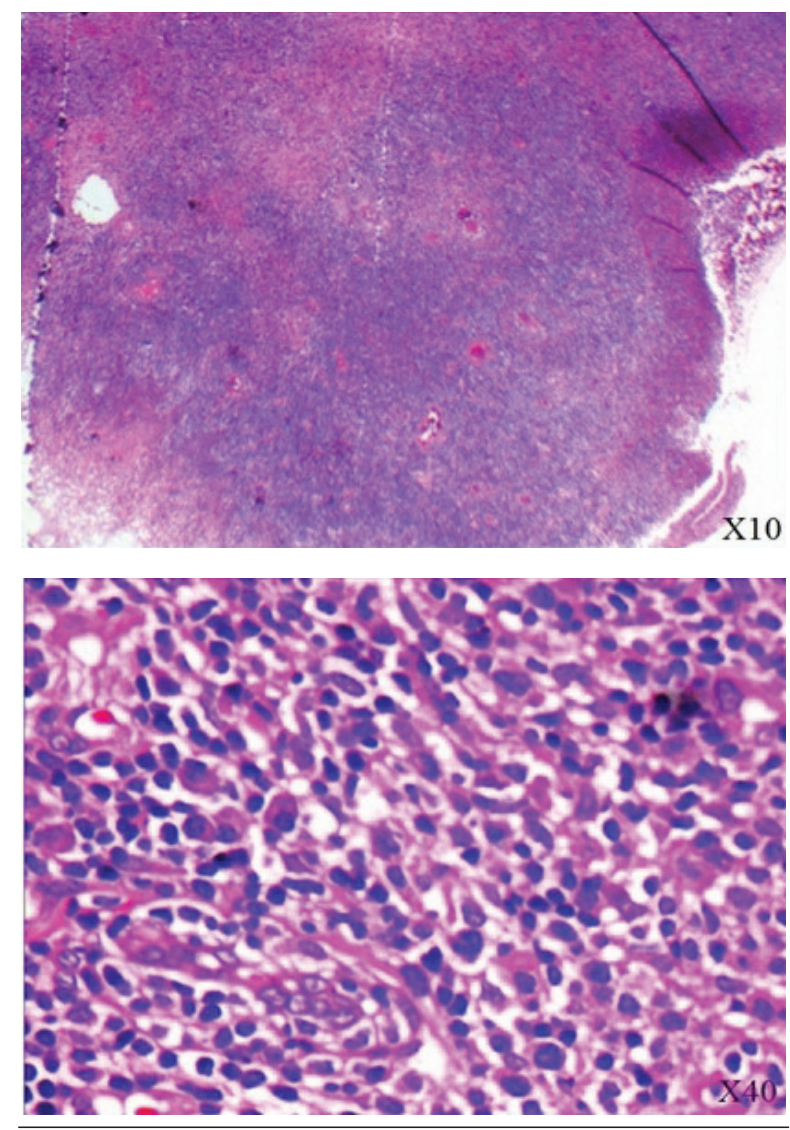

Figure 1: Sections show a diffuse pattern of proliferation of relatively small to medium sized lymphoid cells. The neoplastic cells have scanty cytoplasm with mild nuclear membrane convolution (H\&E X10, X40).
The chromatin was clumped, and nucleoli were inconspicuous. There were some plasma cells and polymorphonuclear cells in the background. The mentioned atypical lymphoid cells were observed in the wall of the vessels and perineural spaces. These cells had T-cell phenotype. They were $\mathrm{CD}^{2+}, \mathrm{CD}^{3+}$, $\mathrm{CD}^{5+}, \mathrm{CD}^{56-}, \mathrm{CD}^{20-}$, and $\mathrm{CD}^{10-}$ (Figure 2).
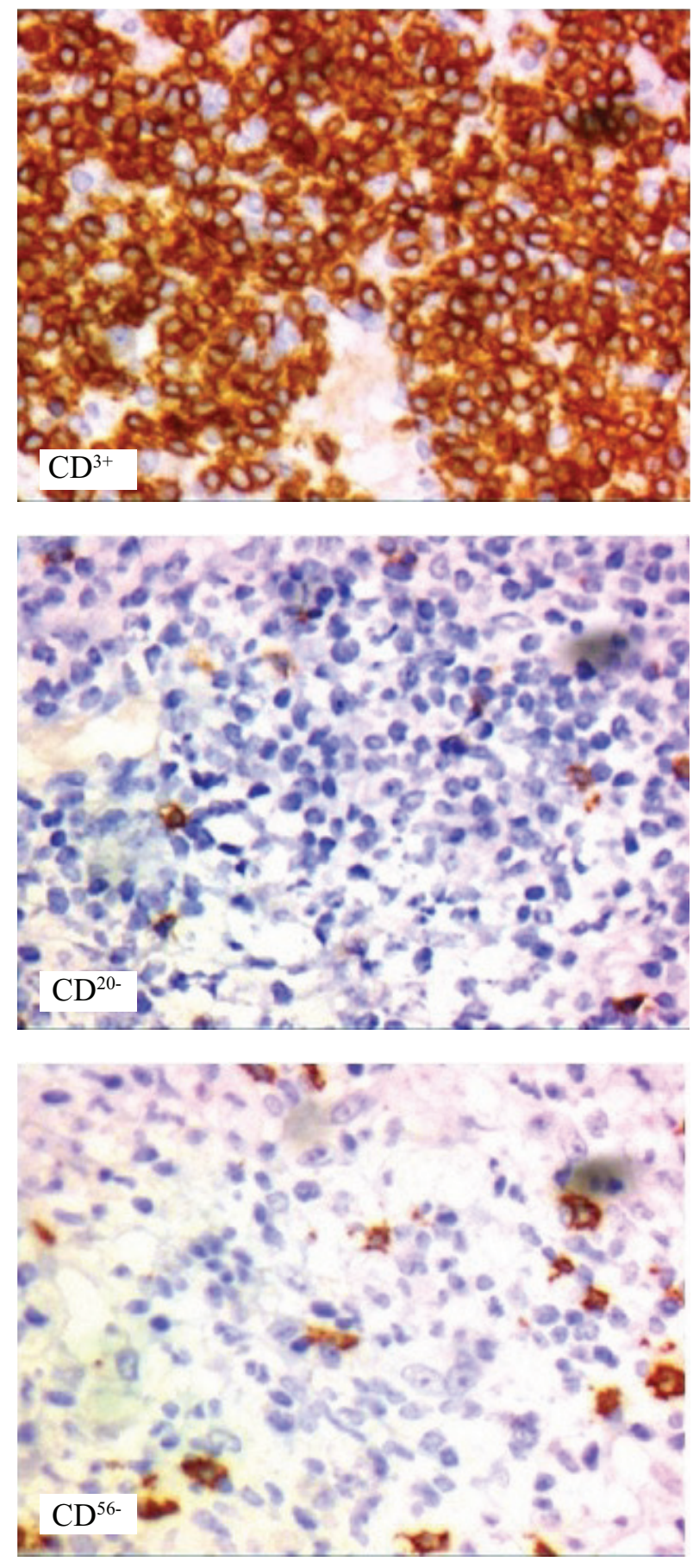

Figure 2: The neoplastic cells are $\mathrm{CD}^{3+}, \mathrm{CD}^{56-}$, and $\mathrm{CD}^{20-}$

These immunohistochemical (IHC) findings were compatible with those of peripheral T-cell lymphoma, not otherwise specified (NOS). The patient had no 
superficial lymphadenopathy or hepatosplenomegaly. Chest computed tomography (CT) scans and abdominal ultrasound did not reveal mediastinal or retroperitoneal lymphadenopathy. There is no positron emission tomography (PET) scan in our city (Yazd); therefore, it could not be performed. Laboratory test results were within normal limits. The result from cerebrospinal fluid and bone marrow aspiration were negative for malignant cells. He was diagnosed with peripheral T-cell lymphoma (PTCL); Ann Arbor stage I. Patient received chemotherapy with CHOEP (cyclophosphamide, Adriamycin, vincristine, etoposide, and prednisone) protocol following which radiotherapy was given. The patient finished chemoradiation therapy. After 15 months of follow-up, the patient is without disease.

\section{DISCUSSION}

Head and neck malignancies contain SCC, nasopharyngeal carcinoma, and malignant lymphomas. Most extranodal lymphomas of the head and neck region involve waldeyer ring. These tumors represent $15 \%-20 \%$ of all lymphomas and approximately $50 \%$ of extranodal lymphomas of the head and neck. Waldeyer ring lymphomas are predominantly NHL of B-cell lineage representing $80 \%$ to $90 \%$ of cases [3]. PTCL does not express $\mathrm{CD}^{56-}$, is rare, and occurs predominantly in the elderly [4]. In contrast to the current case, the disease is usually generalized at the time of diagnosis. A PTCL presenting as an isolated uvular mass is reported by Patel et al., [5]. PTCL has various presentations and can easily be misdiagnosed as other malignant or benign conditions. The current case was presented with progressively worsening odynophagia and sore throat for a period of over six months with uvular enlargement. Walker presented a case of low grade B-cell lymphoma presenting as a uvular mass [6]. A follicular lymphoma of the uvula was described by Kusunoki [7]. Odynophagia and oral discomfort are common symptom, as presented in the current case. In addition, uvular swelling may occur due to infection, allergy, trauma, or tumor. Therefore, the chief complaint in uvular peripheral T-cell lymphoma is nonspecific and diagnosis can be difficult. Differential diagnosis of uvular PTCL includes infectious diseases, reactive lymphoid process, non-healing granulomas, and other lymphoid or non-lymphoid malignancies (SCC and nasopharyngeal carcinoma)
[8]. Negative examinations or biopsy results for the first time are not unusual. Due to these well-known pitfalls, awareness of the clinical history and IHC studies aid in the correct diagnosis [9]. The CHOP regimen (cyclophosphamide, hydroxydaunorubicin, vincristine, and prednisolone) used to treat such patientsimproves theiroutcomes. Radiotherapy alone is effective in the early stage of presentation. Most studies recommend combination of radiotherapy and chemotherapy [10]. The current case received chemotherapy with CHOEP (cyclophosphamide, adriamycin, vincristine, etoposide, and prednisone) protocol following which radiotherapy was given. It is difficult to assess the outcome of PTCL [1].

In conclusion, the current case highlighted the importance of awareness and investigating patients' persistent and progressive symptoms. Clinical knowledge of PTCL and a high index of suspicion are necessary to diagnose this potentially fatal disease.

\section{ACKNOWLEDGEMENTS}

The authors would like to thank the staff of Infectious Diseases Research Center of Yazd Shahid Sadoughi University of Medical Sciences for their kind assistance in performing the study.

\section{CONFLICTS OF INTEREST}

The authors declared no conflict of interest.

\section{ETHICS APPROVAL}

Our work was approved by ethical committee of Shahid Sadoughi University of Medical Sciences, Yazd, Iran.

\section{REFERENCES}

1. Rana K, Narula V, Bhargava EK, Shankar R, Mahajan N. T-cell lymphoma of the oral cavity: case report. J Clin Diagn Res. 2015;9(3):MD03-4. DOI: 10.7860/ JCDR/2015/11883.5690 PMID: 25954642

2. Lai WS, Wang CH, Shih CP. T-cell lymphoma manifesting as a uvular mass. Rheumatology (Oxford). 2013;52(4):608. DOI: $10.1093 /$ rheumatology/kes372 PMID: 23264553

3. Gnepp DR. Diagnostic Surgical Pathology of the Head and Neck E-Book: Expert Consult - Online and Print. 2 ed. Oxford, UK: Elsevier Health Sciences; 2009.

4. Barnes L. Surgical pathology of the head and neck. California, USA: Informa Healthcare; 2009.

5. Patel R, Tomovic S, Kalyoussef E, Eloy JA. Peripheral T-Cell Lymphoma Involving the Uvula. 2013. p. 2.

6. Walker R, Heffelfinger R. Low-grade B-cell lymphoma presenting as a uvular mass. Ear Nose Throat J. 
2012;91(12):E22-4. PMID: 23288827

7. Kusunoki T, Homma H, Kidokoro Y, Yanai A, Wada R, Ikeda K. Primary malignant lymphoma of the uvula. Otorhinolaryngol Head Neck Sur. 2017;2(3):1-2. DOI: 10.15761/ OHNS.1000139

8. Broadfield C, Gilchrist JRM, Pothula V, Ahmad U, Kumar BN. Extranodal NK/T-Cell Lymphoma, Nasal Type (ENKTL) Presenting with Odynophagia. J Surg Open Access. 2016;2(6):1-3. DOI: $10.16966 / 2470-0991.136$
9. Batra P, Shah N, Mathur S. Midline lethal granuloma--a clinical enigma. Indian J Dent Res. 2003;14(3):174-83. PMID: 15164661

10. van der Waal RI, Huijgens PC, van der Valk P, van der Waal I. Characteristics of 40 primary extranodal non-Hodgkin lymphomas of the oral cavity in perspective of the new WHO classification and the International Prognostic Index. Int J Oral Maxillofac Surg. 2005;34(4):391-5. DOI: 10.1016/j.ijom.2004.08.009 PMID: 16053848 